\title{
BUCHBESPRECHUNGEN
}

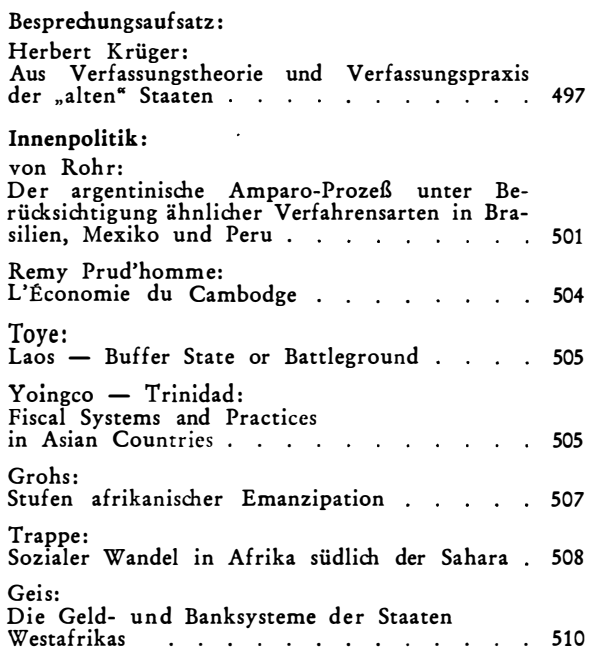

Pauw:

Das Bankwesen in Ostafrika . . . . . . 510

497 Recht:

Fitzgerald:

The Constitutions of Latin America . . . 516

Außenpolitik :

Hoffmann:

Gulliver's Troubles, or the Setting of American

Foreign Policy . . . . . . . . . . . . 517

Kaunda:

A Humanist in Africa . . . . . . . . . 522

Brown:

State and Society in Independant North Africa 524

Weinbuch:

Entkolonisierung und föderales Prinzip im

Spiegel der Französischen Gemeinschaft . . . 525

Völkerrecht :

Sulzbach:

Die Zufälligkeit der Nationen und die Inhalts-

losigkeit der internationalen Politik

\section{AUS VERFASSUNGSTHEORIE UND VERFASSUNGSPRAXIS DER „ALTEN“ STAATEN}

Diese Zeitschrift hat sich als Aufgabe gesetzt, nicht allein die Beschäftigung mit den überseeischen Verfassungen (im weitesten Sinne des Begriffes „Verfassung“). Es geht ihr auch vor allem um die Vergleichung dieser Verfassungen -, etwa mit dem Ziel der Aufdeckung typischer Entwicklungen und deren typische institutionelle (oder vielleicht auch gerade: nichtinstitutionelle) Ergebnisse. Eine solchermaßen umschriebene Vergleichung bliebe aber letztlich doch unergiebig, wenn sie ihre Bemühungen nicht vor den Hintergrund der Verfassungsbildung stellen würde, wie sie den "alten" Staaten einschließlich der Sowjetunion eigen ist - , und sei es auch nur in der Hoffnung, daß hierdurch die Verfassungsentwicklung der "neuen“ Staaten schärfer hervortreten werde. Zur Förderung dieser Seite unseres Vorhabens soll auf diesen Blättern von Zeit zu Zeit über Bücher und Vorgänge berichtet werden, die als solcher Hintergrund für die Verfassungslehre und die Verfassungsvergleichung von Erheblichkeit scheinen.

Die drei Werke, die gemäß einem solchen Programm hier vorgestellt werden sollen, zeichnen sich dadurch aus, daß sie sich über ihren nationalen Gegenstand hinaus zu allgemeinen konstitutionellen Darlegungen erheben, obwohl sich dieser Gehalt in allen Fällen dem Titel nach schwerlich vermuten läßt. Dieser Gehalt ist es, der im folgenden vorgeführt und dadurch einer weiteren Kenntnisnahme zugänglich gemacht werden soll.

Zu Anfang des Jahres 1968 hat der junge schweizerische, in Neuenburg (Neuchâtel) lehrende Staatsrechtler Jean-François A u b e r t einen „Traité de Droit Constitutionell Suisse" (Paris bei Jurisprudence General Dalloz-Neuchâtel bei Editions Ides e Calendes, 1967, 2 Bände VIII + 784 S.) im stattlichen Umfang von 784 Seiten vorgelegt ${ }^{1}$. Für das Grundthema dieser Arbeit, das Schweizerische (Bundes-)Verfassungsrecht, sei lediglich festgestellt, daß es dem Leser ein vollständiges, eingehendes und vor allem sinnhaftes Verständnis vermittelt, das durch eine hervor- 
ragende Darstellung seiner Geschichte von der Helvetik an vertieft und durch ausgedehnte Exkurse in das Verwaltungsrecht (vgl. etwa die Darstellung des Polizeirechtes im Zusammenhang des Problems der Einschränkbarkeit der Grundrechte) und in das Völkerrecht (vgl. z. B. S. 339 ff.) bereichert wird. Einzig die Darstellung des Verhältnisses von Staat und Wirtschaft (S. 664 ff.) läßt Tiefe und Fülle vermissen: Man kann sich des Eindruckes nicht erwehren, daß sie eher 1867 als 1967 geschrieben sein könnte.

Die überpositive Bedeutung des Werkes äußert sich als erstes darin, daß der Verfasser immer wieder die Institute des Schweizer Verfassungsrechts mit den entsprechenden Gebilden fremder Rechtsordnungen vergleicht (etwa S. 231; 259; 179; $282 ; 459 ; 473 ; 628)$ : Leser und vornehmlich Lernende werden auf diese Weise von vornherein von jedem Provinzialismus ferngehalten. $Z$ weitens und vor allem erweist sich als fruchtbringend für die Verfassungsvergleichung die Überzeugung des Verfassers, keine Grundvorstellung des westlichen Verfassungsdenkens verwenden zu dürfen, ohne sie nicht beinahe monographisch dargestellt zu haben. Hiermit erweist sich das Buch als Fundgrube für den Verfassungstheoretiker, ja man kann geradezu von einer, in das positive Verfassungsrecht eingebetteten Gesamtdarstellung der Grundlagen der liberal-demokratischen Verfassungskonzeption sprechen. Zwei Darbietungen scheinen mir in diesem Zusammenhang als Höhepunkte anzusprechen. Es handelt sich einmal um die Beschäftigung (S. 245-274) mit dem selten gestellten Problem, was notwendiger und legitimer Stoff, und zwar allein notwendiger und legitimer Stoff dieses Verfassungsmodells sein kann („Verfassung im materiellen Sinne“ - wozu das in die Schweizer Verfassung aufgenommene Verbot des Absynthes gewiß nicht gehört). Die praktische Bedeutung einer solchen Festlegung der adäquaten Thematik einer Verfassung im eminenten Sinne des Wortes zeigt sich in der Möglichkeit, Klarheit darüber zu gewinnen, wann eine Verfassungsänderung eine solche im materiellen Sinne ist - mit der an sich zwingenden Folgerung, daß es für die Änderung nur formellen Verfassungsrechts der für materielle Änderungen vorgesehenen Solennitäten nicht bedarf (vgl. S. 323-419). Vor allem aber erweist sich als grundsätzlich ergiebig die systematische Analyse des Verfassungsgrundsatzes der Gewaltenteilung (S. 449 ff.). Zusammen mit M. C. J. Vile's, soeben in zweiter Auflage erschienenen geistesgeschichtlichen Untersuchung "Constitutionalism and the Separation of Powers" stellt diese Analyse das beste dar, was man zu diesem öfter beschworenen denn ergründeten Verfassungsgrundsatz heute findet.

Was von der Darstellung der Verfassungsgrundsätze gerühmt werden konnte, gilt nicht minder für Einzelheiten. Als Beispiel hierfür sei genannt die gediegene Erörterung der Frage, ob das Wahlrecht ein subjektives Recht oder eine Funktion sei (S. 412 ff.) -; daß nur das zweite Verständnis dem Modell „Repräsentative Demokratie" gemäß ist, ergibt sich für den Rezensenten zwingend aus der stereotypen Formel, daß der Abgeordnete Vertreter des ganzen Volkes sei: Wie anders sollte der Wähler einen solchen Repräsentanten kreieren können, wenn nicht als Funktionär der Nation?

Pierre Avril's "Le Régime Politique de la Ve République" (Préface de Roger Pinto $-2^{e}$ édition Mise à jour Paris 1967, Librairie Générale de Droit et de Jurisprudence R. Pichon et R. Durand-Anzias = Tome VIII der Bibliothèque Constitutionelle e de Science Politique sous la direction de Georges Burdeau) ist eine überaus scharfe, aber niemals bösartige kritische Darstellung der Verfassung der Fünften Republik Frankreichs und ihrer Handhabung durch de Gaulle und Debré - , eine Kritik, die auch nicht, wie es sonst die Regel zu sein scheint, die Sünden einer liberalen Vergangenheit übergeht, die dem autoritären Regime überhaupt erst eine Chance gegeben hat. Dies alles ist auch nach dem 27. April 1969 noch wichtig genug. Von spezifischem Wert für die Verfassungslehre ist die Arbeit 
wegen ihrer Strategie, Verfassungsnormen und Verfassungspraxis der Fünften Republik im Lichte der Ideale zu kritisieren, die dem Verfasser in beiden Hinsichten vorschweben und die er als Maßstäbe seiner Kritik vortrefflich darstellt —, womit er jedenfalls im Ergebnis eine Verfassungslehre des parlamentarischen Systems liefert, so wie es nach geschriebenen und vor allem auch nach ungeschriebenen Normen sich darstellen sollte. Dieser verfassungstheoretische Ertrag des gründlichen Werkes soll wenigstens in einigen Beispielen vorgeführt werden.

Unter diesem Vorzeichen verdient als erstes Aufmerksamkeit, was der Verfasser im ersten Teil „Le Régime Parlamentaire Assaini?" zum Thema "Gesetz" darlegt. Die Verfassung von 1958 hat die wahrscheinlich unlösbare Frage, welche Art von Regelungen adäquater Inhalt des Gesetzes als Form ist -, und welche Entscheidungen demgemäß in die Zuständigkeit des Parlamentes als Legislative fallen, bemerkenswerterweise nicht wie sonst allgemein üblich offengelassen, sondern durch die Statuierung (Art. 34 II) einer Legaldefinition ausgeräumt, die nicht abstrakt formuliert, sondern konkret aufzählt, m. a. W. eine Generalklausel durch eine Enumeration ersetzt. Alle Regelungen, die demgemäß nicht als Gesetze getroffen werden dürfen, erweisen sich damit ohne weiteres als Sache der Regierung: Ihr wird auf diese Weise eine Fähigkeit zuerkannt, die die deutsche Theorie als „Selbständiges Verordnungsrecht" (selbständig, weil keiner Ermächtigung durch den Gesetzgeber bedürfend) gekannt, aber seit langem verworfen hat ${ }^{2}$. Demgegenüber hält Avril an der Deutung des Gesetzes als "volonté générale" fest. Hiermit wird alles und jedes zum tauglichen Gegenstand eines Gesetzes und damit der parlamentarischen Gesetzgebung erklärt -, vorausgesetzt nur, daß die Allgemeinheit dergleichen will. Die Lösung, für die die Verfassung von 1958 sich entschieden hat, bedeutet daher für Avril "la fin de la suprématie de la loi“ (S. 16) und im Anschluß an P. Durand "Phumiliation du Legislateur". Nicht zuletzt aber hat diese Verschiebung der Gewichte im Verhältnis zwischen Parlament und Regierung auch Gespräche und Zusammenarbeit zwischen diesen beiden Institutionen gestört, wie sie das Parlamentarische System ungeschrieben voraussetzt: Was der Verfasser feinsinnig hierzu zu sagen weiß scheint mir vor allem für die Verfassungslehre von Bedeutung.

Nach solchen tristen Konstatierungen wendet sich der Verfasser im Zeiten Teil des Buches der Exekutive zu. Schon die Überschrift dieses Teiles läßt den Leser ahnen, worauf der Verfasser hinaus will. Sie lautet: "La Dialectique du Pouvoir ou de la Suppléance au Principat“ (S. 163 ff.) -, ein Prinzipat, das später (S. 368) näher charakterisiert wird als „principat paternel“ allerdings weniger im Stil einer Diktatur als dem einer Monarchie. Mit alledem ist angesprochen das Phänomen, das man in Frankreich "personnalisation du pouvoir" nennt (vgl. den Verfasser selbst, S. 34). Die Qualifikation des Staatsoberhauptes im Verständnis von de Gaulle als „principat“ bedeutet etwa: „... l'àdhésion populaire qu'il receuille, est moins une approbation qu'un hommage et une consécration" (S. 327). Was die Mitarbeiter eines solchen princeps angeht, so tritt an die Stelle ihrer Verantwortlichkeit gegenüber Gesetz und Parlament „un système mêlant l'allégeance personelle au chef de l'Etat (die "inconditionelle“ ist - S. 333) à la technicité des spécialistes" (S. 137) -, d. h., die Verpflichtung gegenüber der Person und der Aufgabe, sonst nichts. Diese Personalisierung durch das Prinzipat bringt mit sich Entpolitisierung bis hin zur Apathie (S. 350), ruiniert die Legalität (S. 344) und die Kontinuität (S. 347) und degradiert die Institution (S. 370). Obwohl eine Abwägung zwischen Stärken und Schwächen eines solchen persönlichen Regimentes angekündigt wird (S. 343), ergibt in der Sache die Bilanz jedenfalls gemessen an dem Verfassungsideal, dem der Autor sich verschrieben hat, nur Schwächen.

2 Vgl. S. 30: « Tout ce qui n'est pas expressément rattaché au domaine de la loi formelle, tel que l'énumere l'àrticle 34, est attribué automatiquement est exclusivement au secteur reglementaire, en vertu de l'àrticle 37 •. 
Im Grunde hat der Verfasser ein Organ nur für die klassische parlamentarische Demokratie liberalen Schlages, über deren Sinn- und Arbeitszusammenhänge er Wertvolles zu sagen weiß. Die Frage, ob dieses Modell und seine Ambiance den Gegebenheiten und Anforderungen des ausgehenden zwanzigsten und vor allem dem bevorstehenden einundzwanzigsten Jahrhundert entsprechen, stellt sich ihm nicht. So schärft dieses Werk den verfassungstheoretischen Blick. Verfassungspolitische Anregungen insbesondere auch für die überseeischen Staaten wird man ihm schwerlich entnehmen können.

Während die bisher vorgeführten beiden Bücher zur Erhellung des klassischen liberal-demokratischen Staats- und Verfassungstypus Wesentliches beitragen, ist das nunmehr anzuzeigende Werk wertvoll, weil es diesen Typus zum Gegenstand kritischen Nachdenkens macht. Angesprochen ist hiermit John C. Livingstons und Robert C. Thompsons „The Consent of the Governed" (Second Edition, New York, 1966, The Macmillan Company; XII, 591 S.). Seine beiden Autoren beobachteten „a deep disorder in our political system “ und werten diese Unordnung als "The Crisis of Democratic Politics" (S. 6 ff.). Unter solchen Vorzeichen bieten sie nicht nur eine konzentrierte und eindringliche Schilderung der Verfassungszustände und -verfahren in USA (es handelt sich also trotz des Titels nicht etwa um eine Monographie); sie gehen vielmehr auf deren Schwächen und Unzulänglichkeiten unbefangen und vorurteilslos mit besorgtem Ernst ein. Zwei Erscheinungen sind es, die ihnen vor allem Grund zu solcher Besorgnis geben.

Zum ersten handelt es sich darum, daß das Volk dieser Demokratie politisch nicht dergestalt organisiert ist, wie es das Zusammenspiel zwischen Regierung und Volk erforderte. Dieser Teil der Kritik gilt vor allem den Politischen Parteien. Sie sind in USA, wie allgemein anerkannt ist, nicht mehr als ein lockerer Bund von Vertretern aller möglichen lokalen und provinziellen Belange und Vorurteile (S. 265 ff.; 270 ff.; 282 ff.). Sie sind infolgedessen weder als Regierungs- noch als Oppositionspartei brauchbar -, wie es im übrigen auch mangels einer solchen kontradiktorischen Konstellation nicht zu einer echten parlamentarischen Diskussion kommt (S.14). Dieser Mangel an Zusammenhang und Dauerhaftigkeit zwingt den Präsidenten, von Fall zu Fall Mehrheiten für die von ihm erforderlich gehaltenen, einzelnen Maßnahmen zusammenzubringen ${ }^{3}$. Hiermit werden nicht nur unerhörte Ansprüche an die ohnehin überforderte Arbeitskraft des Präsidenten gestellt. Es wirkt sich beides auch zum Schaden der Sache selbst aus: Eine Mehrheit läßt sich aus Vertretern von Partikularinteressen nur bilden, wenn sie der Präsident durch Abstriche am Gemeininteresse zu gewinnen vermag. $D a$ es scheinbar immer noch eine Tradition gibt, die die Aufgabe wahrer Freiheitsfreunde darin sieht, der Regierung das Leben schwer zu machen (vgl. etwa S. 415), fallen diese Abstriche unter Umständen sehr erheblich aus. Dieses Zurückbeiben von Vorstellungen und Praxis gegenüber den Forderungen des Tages wirkt sich vor allem aus zum Schaden der den USA nach ihrer Auffassung zugefallenen "world leadership" - vgl. z. B. S. 403: "the emergence of the nation as a world leader" $-: \mathrm{Da}$ die Regierung gerade in dieser Hinsicht tragfähige und dauerhafte gesellschaftliche Strukturen nicht vorfindet, entbehrt diese Weltpolitik desjenigen demokratischen Rückhaltes, dessen Fehlen es Dritten schwer macht, sich dieser Führung vorbehaltlos anzuvertrauen.

Der zweite große Gegenstand, dem die Verfasser ihre Besorgnis widmen, betrifft die demokratische Willensbildung. Dem Idealbild nach soll die Allgemeinheit die Trägerin, das Gemeinwohl die Richtschnur dieser Bildung sein. An die Stelle dieses Ideals hat sich eine Wirklichkeit gesetzt, die die Verfasser in allen möglichen Varian-

Entsprechende Beobachtungen über das Fehlen fester und dauerhafter Politischer Parteien und die hieraus
gleichermaßen für den Präsidenten sich ergebenden Schwierigkeiten macht Thomas E. Skidmore für Brasilien; vgl. dessen Politics in Brazil 1930-1964 - An Experiment in Democracy - passim. 
ten über das ganze Buch hin als „brokerage“ beschreiben (vgl. etwa S. 257: „. . the tendency of legislators, to define their role as neutral brokers".). Dieses Leitmotiv der Darstellung meint, daß insbesondere Gesetzgeber und Politiker sich nicht mehr als Repräsentaten der Allgemeinheit und des Gemeinwohles, sondern nur noch als neutrale Vermittler zwischen Sonderinteressen verstehen -, also entsprechend der Diagnose, die die Verfasser für das Parteiwesen gestellt haben: „Congress and state legislatures have tended to become brokerage houses for special interest groups out to gain special favor" (S. 391). Dies gilt aber auch für die Verwaltung, und hier wieder vor allem für die großen unabhängigen Kommissionen wie ICC und FTC, von denen es zum Thema "brokerage politics" heißt (S. 448): "The government often makes no serious effort to regulate; instead, treaties are negotiated between commissioners who serve as the governments ambassadors and the lawyerlobbyists who act as ambassadors for the interests ... And it is highly probable that the protection of the best interests of the people of the state will be identified with the settlement reached by the ambassadors from the attached private interests." Die Feststellung einer solchen Praxis ist nicht neu; bemerkenswert ist jedoch, daß sie in diesem Buch nicht nur beschrieben, sondern verurteilt wird. Diese Verurteilung erhält gesteigertes Gewicht durch die Meinung: „certain combinations of interests are irresistible" (S. 253). Eine der wesentlichen Ursachen aller dieser Úbel sehen die Verfasser in Übereinstimmung mit ihrer grundsätzlichen Kritik am Parteiwesen in dem Fehlen fest und dauerhaft organisierter, straff geführter Politischer Parteien, die imstande wären, solche Interessen abzuweisen oder doch wenigstens im Sinne des Gemeinwohles zu filtern und einzuordnen (vgl. S. 337; 414; 458). Damit wird die Unerläßlichkeit echter Politischer Parteien nicht nur für die Regierung, sondern auch gegenüber der Gesellschaft dargetan: Fehlt dieses letzte und entscheidende Glied in der Kette der Repräsentanten der Gesellschaft zum Staat, dann ergibt sich zweierlei: Die gesellschaftlichen Interessen treten unmittelbar und unverbessert an den Staat heran; und: mangels Stützung durch Politische Parteien vermag sich der Staat allein des Angriffes solcher Interessen nicht zu erwehren. Dies alles gilt um so mehr, wenn, wie die Verfasser meinen, es infolge vorwaltender Gleichgültigkeit an einer unmittelbaren Úberwachung der Politik durch die Allgemeinheit fehlt (S. 458).

Herbert Krüger

Hans Christoph von Rohr

Der argentinische Amparo-Prozeß unter Berücksichtigung ähnlicher Verfahrensarten in Brasilien, Mexiko und Peru

Bonner Rechtswissenschaftliche

Abhandlungen, Bd. 83

Ludwig Röhrscheid Verlag, Bonn, 1969 $184 \mathrm{~S}$.

Wieviele Arbeiten in deutscher Sprache mag es über den Freiheitsschutz „habeas corpus" des angelsächsischen Rechts geben? Auch ohne eine Statistik bei der Hand zu haben, kann man die Behauptung wagen, daß es eine ganze Anzahl sind. Uber sein lateinamerikanisches Pendant, den Amparo-Prozeß, ist dagegen eben erst eine Monographie erschie- nen. Es scheint unglaublich, daß sich nie zuvor die deutsche Rechtsvergleichung ausführlich mit dieser eigensten Rechtsfigur Lateinamerikas befaßt hat. "Die andere Hälfte der Neuen Welt" liegt dermaßen außerhalb des Gesichtsfeldes der deutschen Rechtswissenschaftler, daß jeder Autor, der sich mit dem Recht dieses Gebiets befaßt, einleitend beinahe mit einem Ton der Entschuldigung erklären muß, daß es dort tatsächlich ein studierenswertes Rechtssystem gibt.

Die Arbeit von Hans Christoph v. Rohr hat daher vom Start weg das Verdienst, eine Eintragung mehr in den weißen Flächen dieser "terra nondum cognita" auf den Atlanten der deutschen Rechtsvergleichung zu sein. Schon das würde 